\title{
Book report
}

\section{An "encyclopaedia" for anaesthesia and intensive care medicine: is it possible? \\ Herwig Gerlach}

\begin{abstract}
Professor of Anesthesiology and Intensive Care Medicine, Director, Department of Anesthesiology, Intensive Care and Pain Management, Vivantes, Klinikum Neukoelln, Berlin, Germany
\end{abstract}

Corresponding author: Herwig Gerlach, herwig.gerlach@vivantes.de

Published online: 1 February 2005

Critical Care 2005, 9:E14 (DOI 10.1186/cc3056)

This article is online at http://ccforum.com/content/9/4/E14

(c) 2005 BioMed Central Ltd

Yentis SM, Hirsch NP, Smith GB (Eds): Anaesthesia and Intensive Care Medicine A-Z: An Encyclopaedia of Principles and Practice, 3rd edition. Edinburgh: Butterworth Heinemann; 2004. 564 pp. ISBN 0-7506-8777-0

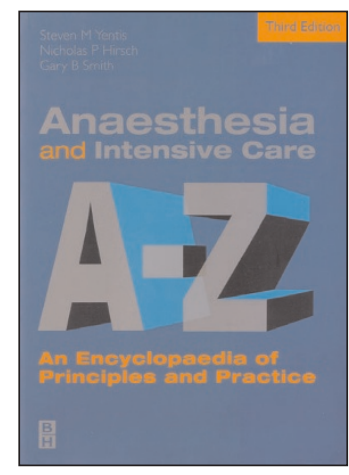

An encyclopaedia is described as 'a work containing exhaustive information on some one branch of knowledge' (Oxford English Dictionary). This is truly a high pretension of Steven Yentis and his co-editors for their new edition of Anaesthesia and Intensive Care Medicine A-Z: An Encyclopaedia of Principles and Practice. According to the Preface, the authors have tried 'to produce a readily accessible source of information' for candidates preparing for the examinations at the Royal College of Anaesthetists. The first edition found acceptance from a broad readership trainees and practitioners, anaesthetists and intensivists. Meanwhile, the content has extended markedly, and the current version has more than 550 pages.

As one leafs through the book, the high quality of the 166 figures and 35 tables is striking. Combining due simplicity and necessary accuracy, the figures present elementary facts about basic anatomy, physiology, pharmacology and specific topics. Within the text, numerous cross-references are of considerable help to the reader in finding related topics and important basic information. The short historical reviews support a better understanding of the complex background. Another feature of the book is the parallelism of scientific basics and practical issues; for example, cartoons and precise descriptions of a chest drainage system or symbols of electrical equipment are presented with the same care as complex plans of the lumbar plexus or the anatomy of the mediastinal tract. This kind of high-quality work has clearly required years of careful analysis, and I am sure that this will be valued by a broad readership.

Some criticisms, however, have to be raised. After thorough review, this book refers predominantly to topics about anaesthesia, whereas intensive care medicine is not always presented in an updated manner. Let us take some examples. The management of severe sepsis and septic shock is not in accordance with current recommendations. Very often, the authors refer to recent (2003) review articles dealing with pathophysiological aspects of the disease. In contrast, none of the major randomized clinical trials from 2001 and 2002, which are part of current guidelines, are cited. Time cannot be the problem, because more recent publications are mentioned. Surprisingly, terms such as 'antithrombin' and 'immunonutrition' are not mentioned. Furthermore, there is no statement regarding immunoglobulins, and no clear recommendation for catecholamine therapy in septic patients is to be found. Finally, recommendations for preventive measures against ventilator-associated pneumonia (such as daily sedation vacation, head-up positioning and low tidal volume) are not mentioned. Taken together, this book is not the ideal source for up-to-date treatment of severely ill patients.

Is this a swan song? Definitely not! I am optimistic that the book will succeed in reaching a broad readership. However, the limitations must be clearly stated. For the young anaesthetist as well as for the practising nurse, this book is a fascinating tool for getting an overview when looking at special items, as it offers both clinical evaluation of therapeutic approaches and basic physiology. For the intensivist, some specific information is definitely missing. In the Preface, the Editors express their hope 'that this book will continue to be useful to all staff who help us care for our patients on a daily basis...' I feel that this is not only a fulfilled hope, but, on the basis of the present result, a fact for which the authors are to be congratulated.

\section{Competing interests}

The author(s) declare that they have no competing interests. 